\title{
Research on the Intelligent Power Communication Network Management Information Model Based on the Web Technology
}

\author{
Qi Xue ${ }^{1, \text { a }}$, Zhirong Liang ${ }^{2, b}$ and Dawang Cheng ${ }^{3, c}$ \\ ${ }^{1}$ Chinese people's Liberation Army Armored Forces Academy, Anhui, 233000, China \\ ${ }^{2}$ Chinese people's Liberation Army Armored Forces Academy, Anhui, 233000, China \\ ${ }^{3}$ Chinese people's Liberation Army Armored Forces Academy, Anhui, 233000, China

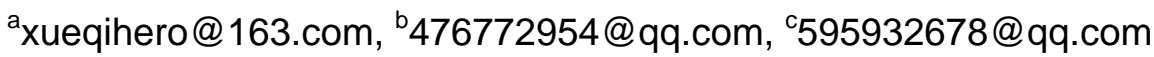

Keywords: Intelligent Power, Communication Network, Management Information Model, Web Technology

\begin{abstract}
With the continuous development of network technology and the addition of network size, speed and complexity, the importance of network management is also increasing. The network management is the process to control a complex computer network so that it has the highest efficiency and productivity. According to the ability of network management systems, this process usually includes data collection, data processing automatically or manually by the manager and then submitted the data to the manager. The construction of intelligent power has increased the dependence of power on information and communication networks, promoted the real-time, security, and other requirements on electricity network.
\end{abstract}

\section{Introduction}

Network management is to monitor and control a complex computer network to ensure its normal operation as long as possible, or when the network fails as quickly as possible to find fault and repair the fault, making it to maximize its effectiveness of the application process . That is, the network management including network monitoring and control two aspects. It is therefore important task NMS is: collection network in the various equipment and systems operating parameters, operational status information; to collect a variety of information to a wide variety of visually presented to the network management personnel; receiving network management personnel instructions or issue control instructions to the network device based on the above information processing result, namely the implementation of network control functions, while monitoring the results of the instruction; and ensure network equipment in accordance with the requirements of NMS work [1].

\section{The Architecture of Network Management}

The architecture of network management is presented by model, which describes the relationship between the NMS and the overall structure of each component of the system as well as between these components. Widely used in network management is the manager / agent model consists of four components [2]:

Management Node. Management node is managed devices can be hosts, routers, switches, hubs, printers, and any other devices with the outside world can communicate status information. Network management agent to run the usual nodes. Agents from the manager of the command or information requests into device-specific instruction-oriented, complete indication of managers, or return it location information of the device. In addition, the agent can also be put events in their own system, proactive notification to the manager. Each agent also maintains a local database to store its state library, historical and affect its operation. .

Management Workstation. Management workstation is actually a computer running special management software. This particular software is the manager, it is on the network to communicate with agents, send commands and receive responses. In this model, most of the computational work 
from the finish, which is to enable the agent to reduce the impact on the equipment as possible. .

MIB. Most of the actual operation of the network uses multiple manufacturers' equipment, in order to manage these workstations can communicate with all devices, information held by such equipment must have a strictly defined standard. This standard is a network management model in the largest part of which details the precise information and the definition format information agent should maintain. In short, each device having one or more variables to describe the state, these variables are known as managed objects, but this object is an object-oriented objects in the system have different meanings, it is only the state, there is no way. All network objects are stored in a place called MIB data structure. .

Management Protocol. Communication management station and the agent uses a management protocol. The agreement allows local object management workstation queries the agent of the state, if necessary, make changes. Network management protocols Operation Command typically include query, set, and three types of notification.

\section{The Research Status of Power Information Communication Network}

Electricity network communication is often a long time, new research projects, many research achievements in this regard. In recent years, some scholars also for the future of smart electricity network communications made to explore and research. Overall, domestic and foreign research status of the power of information and communication networks shows the following characteristics [3]:

Discussion of the power of emerging communications technology, new technology, new architectures are emerging, but the study of smart electricity power era of information and communication networks and the prospect is still in the stage envisaged.

Power Networks as the main content of grid construction and one of the key technologies, has been subject to extensive attention related personnel, whether it is scientific research institutions, power companies or manufacturers of communications equipment, all of the technological development in the field very seriously. In recent years, with the continuous development of power grid construction, continuous advances in information and communication technology, and research to explore the power of communication technology are endless. Currently research is mainly domestic power communication network is still stuck in the simple power communications, the communications network and information network and to increase the power of information and communication network construction is relatively small discussion and study up intelligent power construction increased to a major component content. Researches on intelligent power communication method are still in the vision and the prospect stage.

The demand for electricity Analysis of information and communication based on the current status of major power grids, lack of information and communication needs of the new era of smart electricity demand combined analysis of future

The study technical point of more and less discussion of the power of information and communication network operation mode, but the lack of relevant economic analysis

Studies are based on large domestic purely technical point of view, from a particular communication technology, to explore its application in the realization of the power of information and communication networks, and communication between the different technical features, applications, and many other costs and effectiveness less horizontal comparison and analysis, but the lack of a detailed analysis of smart electricity demand for real communication. The electricity network model based on purely technical point of ICT constructed, often it is "one of the words" a technical field staff, able to meet the electricity needs of ICTs intelligent power era, to be studied.

\section{The Basic Concept of Intelligent Power}

According to the national grid smart proposed power development plan, China's intelligent power construction is "UHV grid based, unified, smooth, efficient, bi-directional communications network connectivity, control, power generation, transmission, substation, distribution, highly 
intelligent electricity and scheduling [4].

Clean. With the research and application of new energy nuclear, wind, solar and other power generation technologies and distributed power, the future of the proportion of clean energy in the final energy consumption will be greatly improved, and thermal power generation in the total proportion of the It will be getting lower and lower, thus greatly reducing energy consumption and pollutant emissions of the power industry. Meanwhile, with the development of control technology and power electronics technology, FACTS UPQC promote the application of technology will greatly eliminate the power grid load side voltage harmonics or flicker phenomenon, so that the full power quality to meet the needs of different users, power will truly be clean, green energy.

Safe. China's future will be the backbone of UHV, EHV using the regional power grid interconnection, etc., to build "a strong unified smart power." Which is our strong focus describe future intelligent power safety. Once the system through a strong network structure, to achieve a strong power transmission capacity and ensure safe and reliable electricity supply; secondary system based on a high degree of redundancy IEC61850 Ethernet technology and dual configuration of system security and other advanced technology, making the ability to resist risks of the grid has been greatly improved, in the event of a terrorist attack or natural disaster, can quickly restore power.

Self-healing. Self-healing means only a small amount or no human intervention, it can achieve grid fault isolation components recover or to minimize or even avoid power interruption to the user. Future intelligent power through real-time online, continuous security assessment and analysis, to achieve the detection of the power element or abnormal operation of local networks, analysis, response and recovery, so as to achieve self-healing grid, reliable and safe operation.

Economic. Intelligent power load forecast in other intelligent analysis technology, the use of advanced intelligent algorithm, electricity consumption, electricity and other basic parameters of the electricity market and network transmission parameters to make more timely and accurate forecasts, through intelligent scheduling system to complete realization various aspects of the power system intelligent scheduling, to avoid blindness generating side. In addition, through advanced energy storage technologies, intelligent power will be able to truly balance power generation and electricity, saving the remaining energy waste, while achieving lower costs of electric power enterprise.

Interactive. Smart power, the client will be deemed complete one of the components of the power system, and management mechanisms through interactive technology and demand response and other user equipment and the behavior of users of electricity in the hair will balance, and other energy saving play an active role. Smart meters will use discretion to consumer energy use by consumers according to their needs to decide when, where and how to use electricity, which will contribute to save electricity, to achieve the intelligent power management, improve the efficiency of energy use increased use of renewable energy, and to make intelligent home devices.

\section{The Web-Based Network Management Information Model}

Traditional network management interface is a network management command-driven remote login screen and must be done by a professional network management staff. Use and maintain NMS requires specialized training of technical personnel, as the network size increases, the complexity of network management functions, the traditional network interface friendliness getting worse. In order to reduce network complexity, lower network management costs, research and development of urgently needed a cross-platform, new network management model easy to use. Web-based network management model can achieve this goal. This new model combines the Web network management functions and network technology, he allows network managers with the same form of www to monitor, manage network systems, you can use the Web browser to easily and quickly configured on any node in the network, control and access to the network and its various parts, this new network management model charm is that it is cross-platform, can solve many interoperability issues arising due to multi-platform structure, which can provide more direct than traditional network management interface, more Easy to use graphical interface (browser operations, and Web pages to www user is very familiar terms), thereby reducing the special requirements for network 
management operations and maintenance personnel. Web-based network management model is a revolution in network management, which will allow users to manage network approach to completely change, so as to achieve "self-managed networks" and "Network management automation" a crucial step [5].

The information model is represented as four levels:

(1) Managers in the browser layer protocol by HITP download APPetl. By JAVA virtual machine can run the downloaded JAVA APPetl program, which can solve cross-platform issues when using applications.

(2) Network Management Center in addition to acting as a Web server, the browser layer also receives a request issued by CORBA, and the request is passed to be able to answer its management agent (sub-administrator) at. Network Management Center also manages a primary database, which is used to store information about the management agent.

(3) Each management agent management subnet, it can be part of a subnet, running on one computer subnet to be independent of the managed subnet. Management agent with a local MBI, the database is used to store data managed network subnet.

(4) Device layer contains the managed devices and each subnet contains a plurality of managed devices.

\section{Conclusions}

Network management has become a very important and necessary part of computer network research and construction, which determines the efficiency and effectiveness of the network resources. The Web-based network management system is a network management system with four-layer structure. The operation costs of intelligent power information and communication network construction are mainly from the network line construction and transformation costs, the device constituting cost of network hardware and software, network operation and management costs, network equipment maintenance and renewal fees. The intelligent power information and communication network is the latest trends and research focus within the current field of network management and has an important practical significance on computer network management and also has a good prospect.

\section{References}

[1] Kai Xie: China Power, Vol. 6 (2004) No 53, p.25-26

[2] Junqing Shuai: China Power Enterprise Management, Vol. 12 (2005) No 27, p.74-76

[3] Peide Sun: Power Information, Vol. 1 (2006) No 33, p.11-14

[4] Hao Sun: Science and Technology Forum, Vol. 3 (2007) No33, p.121-124

[5] Xinyu Du: Electric Power Magazine, Vol. 12 (2010) No5, p.85-90 